\title{
QCD effects in Higgs boson production at hadron colliders
}

\author{
Massimiliano Grazzini* \\ INFN, Sezione di Firenze, I-50019, Sesto Fiorentino, Florence, Italy \\ and \\ Institute for Theoretical Physics, ETH Zurich, 8093 Zurich, Switzerland \\ E-mail: grazzini@fi.infn.it
}

We present updated predictions for Higgs boson production at the Tevatron and the LHC and we review their corresponding uncertainties. We report on a study of the impact of QCD radiative corrections on the Higgs boson search at the Tevatron.

RADCOR 2009 - 9th International Symposium on Radiative Corrections (Applications of Quantum Field Theory to Phenomenology),

October 25 - 302009

Ascona, Switzerland

\footnotetext{
*Speaker.
} 


\section{Introduction}

The discovery of the Standard Model (SM) Higgs boson, or its equivalent in theories beyond the Standard Model, is one of the main goals of the experimental program of high-energy colliders. At the LHC the Higgs boson can be discovered over the full mass range up to $m_{H} \sim 1 \mathrm{TeV}$ within a few years of running. At the Tevatron, the $\mathrm{CDF}$ and $\mathrm{D} 0$ experiments are now sensitive to a Higgs signal at $m_{H} \sim 165 \mathrm{GeV}[1]$.

The dominant mechanism for SM Higgs boson production at hadron colliders is gluon-gluon fusion, through a heavy-quark (mainly, top-quark) loop. The dynamics of this process is controlled by strong interactions, and thus studies of the effect of QCD radiative corrections are necessary to obtain accurate theoretical predictions.

In QCD perturbation theory the leading order (LO) contribution to the $g g \rightarrow H$ cross section is proportional to $\alpha_{\mathrm{S}}^{2}, \alpha_{\mathrm{S}}$ being the QCD coupling. The QCD corrections have been computed at next-to-leading order (NLO) [2,3] in the heavy-top limit, and with full dependence on the masses of the top and bottom quarks [3]. Next-to-next-to-leading order (NNLO) corrections have been obtained in the heavy-top limit [4]. These QCD corrections, which are dominated by radiation of soft and virtual gluons [5], lead to a substantial increase of the LO result. The QCD computation up to NNLO has been consistently improved by adding the resummation of soft-gluon logarithmic contributions, up to next-to-next-to-leading logarithmic (NNLL) accuracy [6].

Recent years have also seen a substantial progress in the computation of radiative corrections to more exclusive observables $[7,8,9,10]$. In particular, in the case of Higgs boson production, two independent fully exclusive NNLO computations are now available $[8,9]$.

This contribution is divided in two parts. In the first part we discuss an update for the total cross section [11], and review the corresponding uncertainties. In the second part we consider the fully exclusive NNLO calculation, and report on a study [12] of the impact of QCD radiative corrections on the Higgs boson search at the Tevatron.

\section{Total cross section}

In this Section we present an update [11] of the NNLL+NNLO computation of Ref. [6]. The results are obtained using the MSTW2008 NNLO partons [13]. We first consider the top-quark contribution in the loop, and perform the calculation up NNLL+NNLO in the large- $m_{t}$ limit. The result is rescaled by the exact $m_{t}$ dependent Born cross section: recent work has definitely shown that this procedure provides an excellent approximation (to better than $1 \%$ for $m_{H} \lesssim 300 \mathrm{GeV}$ ) of the exact top-quark contribution [14]. We then consider the bottom-quark contribution. Since in this case the effective theory approach is not applicable, we follow Ref. [15] and we include this contribution up to NLO only [3]. Finally, we correct the result by including the EW effects [16] as evaluated in Ref. [17]. Our central predictions $\left(\sigma^{\text {best }}\right)$ are obtained by setting the factorization $\left(\mu_{F}\right)$ and renormalization $\left(\mu_{R}\right)$ scales equal to the Higgs boson mass. Our results for the Tevatron and the $\mathrm{LHC}(\sqrt{s}=14 \mathrm{TeV})$ are presented in Tables 1 and 2, respectively. Comparing to our previous predictions (see Tables 1 and 2 of Ref. [6]), the cross sections change significantly. At the Tevatron the effect ranges from $+9 \%$ for $m_{H}=115 \mathrm{GeV}$ to $-9 \%$ for $m_{H}=200 \mathrm{GeV}$. At the LHC the effect 
goes from $+30 \%$ for $m_{H}=115 \mathrm{GeV}$ to $+9 \%$ for $m_{H}=300 \mathrm{GeV}$, the increase being mainly due to the new MSTW2008 PDFs.

The calculation discussed above is now available through an online calculator [18], that can be used to reproduce the results of Tables 1 and 2 or to repeat the calculation for different Higgs boson masses and/or collider energies.

Our results for the Tevatron can be compared to those presented in Ref. [15], obtained using the same set of PDFs. This computation includes an estimate of mixed QCD-EW contributions. The main difference with our work arises in the calculation of the top-quark contribution to the cross section. In Ref. [15] the latter contribution is computed up to NNLO but choosing $\mu_{F}=\mu_{R}=m_{H} / 2$, as an attempt to mimic the effects of soft-gluon resummation beyond NNLO. The final numerical differences at the Tevatron turn out to be small and of the order of a few per mille at the lowest masses, increasing to $2.5 \%$ at $m_{H}=200 \mathrm{GeV}$.

The NNLL+NNLO calculation discussed above could be improved in various respects. Logarithmically enhanced terms beyond NNLL from the Sudakov exponent have been evaluated in Ref. [19, 20]. Their effect, when combined with a full $\mathrm{N}^{3} \mathrm{LO}$ calculation, can lead to a reduction of scale uncertainties to about 5\% [19]. The exact small- $x$ behavior of the NNLO coefficient function is also known [21, 22] and could be included. The numerical effect is, however, smaller than $1 \%$ for a light Higgs. By contrast, the uncertainty that affects the Higgs production cross section is still large. The uncertainty basically has two origins: the one coming from the partonic cross sections, and the one arising from our limited knowledge of the PDFs.

Uncalculated higher-order QCD radiative corrections are the most important source of uncertainty on the partonic cross section, and are estimated through scale variations. The scale uncertainty of our results (see Tables 1 and 2) is about $\pm 9-10 \%$ at the Tevatron and ranges from about $\pm 10 \%\left(m_{H}=110 \mathrm{GeV}\right)$ to about $\pm 7 \%\left(m_{H}=300 \mathrm{GeV}\right)$ at the LHC. We note that the effect of scale variations in our resummed calculation is considerably reduced with respect to the corresponding NNLO result. The reduction is more sizeable at the Tevatron, where the resummation effect is more important.

The other important source of uncertainty in the cross section is the one coming from PDFs. The MSTW2008 NNLO set provides 40 different grids that allow us to evaluate the experimental uncertainties. The outcoming uncertainties (at 90\% CL) are reported in Tables 1 and 2. At the Tevatron the effect ranges from $\pm 6 \%\left(m_{H}=115 \mathrm{GeV}\right)$ to about $\pm 10 \%\left(m_{H}=200 \mathrm{GeV}\right)$, while at the LHC it is about $\pm 3 \%$ in the mass range we have considered.

A related and important uncertainty is the one coming from the value of the QCD coupling. Higgs production through gluon fusion starts at $\mathscr{O}\left(\alpha_{\mathrm{S}}^{2}\right)$ and thus this uncertainty is expected to have a relevant role. Recently the MSTW collaboration has studied the combined effect of PDF $+\alpha_{\mathrm{S}}$ uncertainties [23]. We find that at the LHC the PDF+ $\alpha_{\mathrm{S}}$ uncertainty is about $7 \%$ at $90 \% \mathrm{CL}$ $\left(m_{H} \leq 300 \mathrm{GeV}\right)$, whereas at the Tevatron it ranges from 7 to $18 \%\left(m_{H} \leq 200\right)$. In particular, for $m_{H}=165 \mathrm{GeV}$, we get at the Tevatron $\sigma_{\mathrm{best}}=0.389 \mathrm{pb}_{-7.7 \%}^{+9.2 \%}(\mathrm{scale})_{-10.1 \%}^{+13.2 \%}\left(\alpha_{\mathrm{S}}+\mathrm{PDF} @ 90 \% \mathrm{CL}\right)$.

We finally point out that, besides MSTW, we have at present only two other NNLO parton analyses: ABKM09 [24] and JR09VFNNLO [25]. A comparison of the central values of the cross section shows that at the LHC ABKM09 (JR09VFNNLO) result is smaller than MSTW by about $7 \%(11 \%)$ for $m_{H}=115 \mathrm{GeV}$ and by $11 \%(8 \%)$ for $m_{H}=300 \mathrm{GeV}$. At the Tevatron ABKM09 (JR09VFNNLO) result is smaller than MSTW by about $26 \%$ (2\%) for $m_{H}=165 \mathrm{GeV}$. 


\begin{tabular}{|c|c|c|c|}
\hline$m_{H}$ & $\sigma^{\text {best }}$ & Scale & $\mathrm{PDF}$ \\
\hline 100 & 1.861 & $\begin{array}{l}+0.192 \\
-0.174\end{array}$ & ${ }_{-0.101}^{+0.094}$ \\
\hline 105 & 1.618 & ${ }_{-0.149}^{+0.165}$ & ${ }_{-0.09}^{+0.08}$ \\
\hline 110 & 1.413 & ${ }_{-0.127}^{+0.142}$ & ${ }_{-0.083}^{+0.077}$ \\
\hline 115 & 1.240 & ${ }_{-0.110}^{+0.123}$ & ${ }_{-0.075}^{+0.070}$ \\
\hline 120 & 1.093 & $\begin{array}{l}+0.100 \\
-0.095\end{array}$ & ${ }_{-0.06}^{+0.06}$ \\
\hline 125 & 0.967 & $\begin{array}{l}+0.094 \\
-0.083\end{array}$ & $\begin{array}{l}+0.059 \\
{ }_{-0.063}\end{array}$ \\
\hline 130 & 0.858 & $\begin{array}{l}+0.082 \\
-0.072\end{array}$ & $\begin{array}{l}{ }^{+0.054} \\
-0.058\end{array}$ \\
\hline
\end{tabular}

\begin{tabular}{|c|c|c|c|}
\hline$m_{H}$ & $\sigma^{\text {best }}$ & Scale & PDF \\
\hline 135 & 0.764 & $\begin{array}{l}+0.073 \\
-0.063\end{array}$ & $\begin{array}{l}+0.050 \\
-0.053\end{array}$ \\
\hline 140 & 0.682 & $\begin{array}{l}+0.065 \\
-0.056\end{array}$ & $\begin{array}{l}{ }_{-0.049}^{+0.046} \\
\end{array}$ \\
\hline 145 & 0.611 & $\begin{array}{l}+0.057 \\
-0.049\end{array}$ & $\begin{array}{l}{ }_{-0.045}^{0} \\
-0.042\end{array}$ \\
\hline 150 & 0.548 & $\begin{array}{l}+0.051 \\
-0.044\end{array}$ & $\begin{array}{l}{ }_{-0.042}^{0.039} \\
-0.039\end{array}$ \\
\hline 155 & 0.492 & $\begin{array}{l}+0.045 \\
-0.039\end{array}$ & $\begin{array}{l}+0.036 \\
-0.038\end{array}$ \\
\hline 160 & 0.439 & $\begin{array}{l}+0.040 \\
-0.034\end{array}$ & $\begin{array}{l}+0.033 \\
-0.035\end{array}$ \\
\hline 165 & 0.389 & $\begin{array}{l}+0.036 \\
-0.030\end{array}$ & $\begin{array}{l}+0.030 \\
-0.032\end{array}$ \\
\hline
\end{tabular}

\begin{tabular}{|c|c|c|c|}
\hline$m_{H}$ & $\sigma^{\text {best }}$ & Scale & PDF \\
\hline 170 & 0.349 & $\begin{array}{l}+0.03 \\
-0.027\end{array}$ & $\begin{array}{l}+0.028 \\
-0.029\end{array}$ \\
\hline 175 & 0.314 & $\begin{array}{l}+0.029 \\
-0.024\end{array}$ & $\begin{array}{l}+0.026 \\
-0.027\end{array}$ \\
\hline 180 & 0.283 & $\begin{array}{l}{ }_{-0.021}^{+0.026} \\
\end{array}$ & $\begin{array}{l}{ }_{-0.025}^{+0.024} \\
\end{array}$ \\
\hline 185 & 0.255 & $\begin{array}{l}{ }_{-0.019}^{+0.023} \\
{ }_{0}\end{array}$ & $\begin{array}{l}{ }_{-0.023}^{+0.022} \\
\end{array}$ \\
\hline 190 & 0.231 & $\begin{array}{l}+0.021 \\
-0.017\end{array}$ & $\begin{array}{l}{ }_{-0.021}^{+0.020} \\
{ }_{-0.020}\end{array}$ \\
\hline 195 & 0.210 & $\begin{array}{l}+0.019 \\
{ }_{-0.015}^{+0.9}\end{array}$ & $\begin{array}{l}+0.019 \\
{ }_{-0.020}^{+0.019}\end{array}$ \\
\hline 200 & 0.192 & $\begin{array}{l}+0.017 \\
-0.014\end{array}$ & $\begin{array}{l}+0.018 \\
{ }_{-0.019}^{+}\end{array}$ \\
\hline
\end{tabular}

Table 1: Cross sections (in pb) at the Tevatron $\left(\mu_{F}=\mu_{R}=m_{H}\right)$ with $\sqrt{s}=1.96 \mathrm{TeV}$ using the MSTW2008 [13] parton densities.

\begin{tabular}{|c|c|c|c|}
\hline$m_{H}$ & $\sigma^{\text {best }}$ & Scale & PDF \\
\hline 100 & 74.58 & $\begin{array}{l}+7.18 \\
-7.54\end{array}$ & $\begin{array}{l}+1.86 \\
-2.45\end{array}$ \\
\hline 110 & 63.29 & $\begin{array}{l}+5.87 \\
-6.20\end{array}$ & $\begin{array}{l}+1.54 \\
-2.02\end{array}$ \\
\hline 120 & 54.48 & $\begin{array}{l}+4.88 \\
-5.18\end{array}$ & $\begin{array}{l}+1.30 \\
-1.70\end{array}$ \\
\hline 130 & 47.44 & $\begin{array}{l}+4.12 \\
-4.38\end{array}$ & $\begin{array}{l}+1.12 \\
-1.45\end{array}$ \\
\hline 140 & 41.70 & $\begin{array}{l}+3.47 \\
-3.75\end{array}$ & $\begin{array}{l}+0.97 \\
-1.25\end{array}$ \\
\hline 150 & 36.95 & $\begin{array}{l}+3.02 \\
-3.24\end{array}$ & $\begin{array}{l}+0.85 \\
-1.10\end{array}$ \\
\hline 160 & 32.59 & $\begin{array}{l}+2.60 \\
-2.79\end{array}$ & $\begin{array}{l}+0.73 \\
-0.97\end{array}$ \\
\hline
\end{tabular}

\begin{tabular}{|c|c|c|c|}
\hline$m_{H}$ & $\sigma^{\text {best }}$ & Scale & PDF \\
\hline 170 & 28.46 & $\begin{array}{l}+2.22 \\
-2.39\end{array}$ & $\mid \begin{array}{l}+0.65 \\
-0.84\end{array}$ \\
\hline 180 & 25.32 & $\begin{array}{l}+1.92 \\
-2.08\end{array}$ & $\begin{array}{l}+0.58 \\
-0.74\end{array}$ \\
\hline 190 & 22.63 & $\begin{array}{l}+1.68 \\
-1.83\end{array}$ & $\begin{array}{l}+0.52 \\
-0.66\end{array}$ \\
\hline 200 & 20.52 & $\begin{array}{l}+1.49 \\
-1.63\end{array}$ & $\begin{array}{l}+0.48 \\
-0.60\end{array}$ \\
\hline 210 & 18.82 & $\begin{array}{l}+1.34 \\
-1.47\end{array}$ & $\begin{array}{l}+0.45 \\
-0.55\end{array}$ \\
\hline 220 & 17.38 & $\begin{array}{l}+1.22 \\
-1.33\end{array}$ & $\begin{array}{l}+0.42 \\
-0.51\end{array}$ \\
\hline 230 & 16.15 & ${ }_{-1.22}^{+1.11}$ & $\begin{array}{l}+0.39 \\
-0.48\end{array}$ \\
\hline
\end{tabular}

\begin{tabular}{|c|c|c|c|}
\hline$m_{H}$ & $\sigma^{\text {best }}$ & Scale & PDF \\
\hline 240 & 15.10 & $\begin{array}{l}+1.03 \\
-1.12\end{array}$ & $\begin{array}{l}+0.37 \\
-0.45\end{array}$ \\
\hline 250 & 14.19 & $\begin{array}{l}+0.95 \\
-1.04\end{array}$ & ${ }_{-0.43}^{+0.36}$ \\
\hline 260 & 13.41 & $\begin{array}{l}+0.88 \\
{ }_{-0}^{+0.97}\end{array}$ & $\begin{array}{l}+0.35 \\
-0.41\end{array}$ \\
\hline 270 & 12.74 & $\begin{array}{l}+0.83 \\
-0.91\end{array}$ & $\begin{array}{l}+0.33 \\
-0.39\end{array}$ \\
\hline 280 & 12.17 & $\begin{array}{l}+0.78 \\
-0.86\end{array}$ & $\begin{array}{l}+0.33 \\
-0.38\end{array}$ \\
\hline 290 & 11.71 & $\begin{array}{l}{ }_{-0.82}^{+0.74} \\
{ }_{-0.84}\end{array}$ & $\begin{array}{l}+0.32 \\
-0.37\end{array}$ \\
\hline 300 & 11.34 & $\begin{array}{l}+0.71 \\
{ }_{-0.78}\end{array}$ & $\begin{array}{l}+0.32 \\
-0.36\end{array}$ \\
\hline
\end{tabular}

Table 2: Cross sections (in pb) at the LHC $\left(\mu_{F}=\mu_{R}=m_{H}\right)$ with $\sqrt{s}=14 \mathrm{TeV}$ using the MSTW2008 [13] parton densities. 
Although these three NNLO sets are obtained through different approaches, the large differences in the corresponding results confirm that the uncertainty in the total Higgs production cross section is still large and, at least at the Tevatron, dominated by PDFs.

\section{QCD effects in $H \rightarrow W W \rightarrow l v l v$ at the Tevatron}

In the previous Section we have discussed in detail perturbative predictions for the fully inclusive Higgs production cross section. Total cross sections, however, are ideal quantities: experiments have always a finite acceptance. To properly take into account the kinematical cuts applied in the experimental analysis, fully exclusive calculations are actually needed. LO calculations are in this respect straightforward: one can compute the relevant matrix element and integrate it numerically over the multiparton phase space. Beyond LO the QCD computation is affected by infrared singularities that prevent a straightforward implementation of numerical techniques.

In particular, at NNLO, only few fully exclusive computations exist, due to their substantial conceptual and technical complications [7, 8, 9, 10]. For Higgs boson production through gluon fusion, two fully independent computations exist that are implemented in available numerical codes $[8,9]$.

The CDF and D0 collaborations at the Tevatron have recently reported a combination of their results up to $5.4 \mathrm{fb}^{-1}$. According to this combination, a SM Higgs boson of masses between 163 and $166 \mathrm{GeV}$ is excluded at $95 \% \mathrm{CL}$. In this mass region the signal is dominated by the $H \rightarrow W W \rightarrow l v l v$ channel, which provides a small number of events over a huge background. After a first cut-based selection, background processes remain dominant and processing of real data and Monte-Carlo simulations with Artificial Neural Network (ANN) methods follows. Given the sensitivity of the gluon fusion cross-section to higher order effects, it is important to establish that the sophisticated methods used in the Tevatron analysis account for these effects within the estimated uncertainties.

In Ref.[12] we have performed a study of the inpact of cuts on the Higgs boson signal. We assume a Higgs boson mass $m_{H}=160 \mathrm{GeV}$ and apply the following cuts. We consider the $H \rightarrow$ $W W \rightarrow \mu^{+} \mu^{-} \nu \bar{v}$ channel and require at least one lepton with $p_{T}>20 \mathrm{GeV}$ and $|\eta|<1.1$. The invariant mass of the charged leptons should be $m_{l l}>16 \mathrm{GeV}$. Leptons should be isolated: the total transverse energy in a cone of radius $\mathrm{R}=0.4$ should be smaller than $10 \%$ of the lepton $p_{T}$. Jets are defined according to the $k_{T}$ algorithm with $D=0.4$ : a jet is required to have $p_{T}>15 \mathrm{GeV}$ and $|\eta|<3$.

We define the variable MET* $^{*}$ as

$$
\mathrm{MET}^{*}=\left\{\begin{array}{l}
\text { MET } \quad, \phi \geq \pi / 2 \\
\operatorname{MET} \times \sin \phi, \phi<\pi / 2
\end{array},\right.
$$

where $\phi$ is the angle in the transverse plane between the missing transverse energy, MET, and the nearest charged lepton or jet. We require $\mathrm{MET}^{*}>25 \mathrm{GeV}$, which suppresses the background from Drell-Yan lepton pairs and removes contributions from mismeasured leptons or jets. To suppress the $t \bar{t}$ background, we require at most one hadronic jet.

With the cuts discussed above the NLO K-factor is reduced from 2.41 to 2.15 and the NNLO $\mathrm{K}$-factor is reduced from 3.31 to 2.59 (for $\mu_{F}=\mu_{R}=m_{H}$ ). In order to study the stability of pertur- 
bative corrections in the presence of these cuts we have studied a set of kinematical distributions that can be computed with our NNLO programs. We consider the transverse momenta of the leading and trailing lepton $p_{\mathrm{T}}^{\text {hard }}, p_{\mathrm{T}}^{\text {soft }}$, the invariant mass of the charged lepton, $m_{l l}$, the azimuthal separation of the charged leptons in the transverse plane and the missing transverse energy, MET.

A study of these distributions up to NNLO does not show significant instabilities. We have then compared the results to the same distributions obtained with PYTHIA and MC@NLO, rescaled so as to match the total NNLO cross section, without finding significant differences.

An essential part of the experimental studies concerns distributions of discrimination variables, defined via ANNs. In Ref. [12] we have studied for the first time an ANN output distribution up to NNLO in perturbation theory, using as input variables the leptonic quantities defined above. The results confirm the agreement discussed above.

Despite the agreement in the shape of the leptonic distributions, confirmed by the ANN analysis, a comparison of the acceptances show some discrepancy. The acceptance obtained with HERWIG and MC@NLO is consistent with the acceptance from the NNLO calculation. In contrast, we find that the acceptance computed with PYTHIA is between $12 \%$ and $21 \%$ smaller than the NNLO acceptance, depending on the choice of the renormalization and factorization scales. This result is not significantly altered by hadronization and underlying event effects and appears instead to be related to the matrix element and parton shower implementation in PYTHIA itself.

\section{Summary}

We have presented updated predictions for Higgs boson production at the Tevatron and the LHC, and discussed their uncertainties. We have presented a study of the impact of QCD radiative corrections on the Higgs search in the $H \rightarrow W W \rightarrow l v l v$ channel at the Tevatron, based on the NNLO calculations of Refs. [8, 9]. This study shows that these NNLO programs can provide an essential help in the validation of the results from standard Monte Carlo event generators and in the assessment of theoretical uncertainties.

\section{Acknowledgements}

I would like to thank the organizers of RADCOR 2009 for their kind invitation and for financial support.

\section{References}

[1] Tevatron Electroweak Working Group and CDF Collaboration and D0 Collaboration, report FERMILAB-CONF-09-557-E, arXiv:0911.3930.

[2] S. Dawson, Nucl. Phys. B 359 (1991) 283.

[3] A. Djouadi, M. Spira and P. M. Zerwas, Phys. Lett. B 264 (1991) 440; M. Spira, A. Djouadi, D. Graudenz and P. M. Zerwas, Nucl. Phys. B 453 (1995) 17.

[4] R. V. Harlander and W. B. Kilgore, Phys. Rev. Lett. 88 (2002) 201801; C. Anastasiou and K. Melnikov, Nucl. Phys. B 646 (2002) 220; V. Ravindran, J. Smith and W. L. van Neerven, Nucl. Phys. B 665 (2003) 325. 
[5] M. Kramer, E. Laenen and M. Spira, Nucl. Phys. B 511 (1998) 523; S. Catani, D. de Florian and M. Grazzini, JHEP 0105 (2001) 025; R. V. Harlander and W. B. Kilgore, Phys. Rev. D 64 (2001) 013015 .

[6] S. Catani, D. de Florian, M. Grazzini and P. Nason, JHEP 0307 (2003) 028.

[7] A. Gehrmann-De Ridder, T. Gehrmann, E. W. N. Glover and G. Heinrich, Phys. Rev. Lett. 99, 132002 (2007), JHEP 0712, 094 (2007), Phys. Rev. Lett. 100, 172001 (2008), JHEP 0905 (2009) 106; S. Weinzierl, Phys. Rev. Lett. 101, 162001 (2008), JHEP 0906 (2009) 041 JHEP 0907 (2009) 009, Phys. Rev. D 80 (2009) 094018.

[8] C. Anastasiou, K. Melnikov and F. Petriello, Phys. Rev. Lett. 93 (2004) 262002, Nucl. Phys. B 724 (2005) 197; C. Anastasiou, G. Dissertori and F. Stockli, JHEP 0709 (2007) 018.

[9] S. Catani and M. Grazzini, Phys. Rev. Lett. 98 (2007) 222002; M. Grazzini, JHEP 0802 (2008) 043.

[10] K. Melnikov and F. Petriello, Phys. Rev. Lett. 96, 231803 (2006), Phys. Rev. D 74, 114017 (2006); S. Catani, L. Cieri, G. Ferrera, D. de Florian and M. Grazzini, Phys. Rev. Lett. 103 (2009) 082001.

[11] D. de Florian and M. Grazzini, Phys. Lett. B 674 (2009) 291.

[12] C. Anastasiou, G. Dissertori, M. Grazzini, F. Stockli and B. R. Webber, JHEP 0908 (2009) 099.

[13] A. D. Martin, W. J. Stirling, R. S. Thorne and G. Watt, Eur. Phys. J. C 63 (2009) 189.

[14] R. V. Harlander and K. J. Ozeren, JHEP 0911 (2009) 088; A. Pak, M. Rogal and M. Steinhauser, arXiv:0911.4662.

[15] C. Anastasiou, R. Boughezal and F. Petriello, JHEP 0904 (2009) 003.

[16] U. Aglietti, R. Bonciani, G. Degrassi and A. Vicini, Phys. Lett. B 595 (2004) 432; G. Degrassi and F. Maltoni, Phys. Lett. B 600 (2004) 255; U. Aglietti, R. Bonciani, G. Degrassi and A. Vicini, contributed to the TeV4LHC Workshop, Brookhaven, Upton, New York, february 2005, arXiv:hep-ph/0610033.

[17] S. Actis, G. Passarino, C. Sturm and S. Uccirati, Phys. Lett. B 670 (2008) 12, Nucl. Phys. B 811 (2009) 182.

[18] http://theory.fi.infn.it/grazzini/hcalculators.html

[19] S. Moch and A. Vogt, Phys. Lett. B 631 (2005) 48.

[20] E. Laenen and L. Magnea, Phys. Lett. B 632 (2006) 270; A. Idilbi, X. d. Ji, J. P. Ma and F. Yuan, Phys. Rev. D 73 (2006) 077501; V. Ravindran, Nucl. Phys. B 746 (2006) 58, Nucl. Phys. B 752 (2006) 173.

[21] S. Marzani, R. D. Ball, V. Del Duca, S. Forte and A. Vicini, Nucl. Phys. B 800 (2008) 127, Nucl. Phys. Proc. Suppl. 186 (2009) 98.

[22] R. V. Harlander, H. Mantler, S. Marzani and K. J. Ozeren, arXiv:0912.2104.

[23] A. D. Martin, W. J. Stirling, R. S. Thorne and G. Watt, Eur. Phys. J. C 64 (2009) 653 [arXiv:0905.3531 [hep-ph]].

[24] S. Alekhin, J. Blumlein, S. Klein and S. Moch, arXiv:0908.2766 [hep-ph].

[25] P. Jimenez-Delgado and E. Reya, Phys. Rev. D 80 (2009) 114011 [arXiv:0909.1711 [hep-ph]]. 